\title{
Does Decentralization Reform Promote Local Economic Growth? A Meta-Analysis
}

\author{
Songhao $\mathrm{Yu}^{1}$, Maoxuan Wang ${ }^{1}$ and Jinsong Meng ${ }^{1, *}$ \\ ${ }^{1}$ School of Government, Beijing Normal University, Beijing, 100875, China \\ *Corresponding author. Email: 201831260004@mail.bnu.edu.cn
}

\begin{abstract}
Although some theories believe that decentralization reform can promote local economic growth, the empirical evidence in this research field shows strong inconsistency. This article conducted a meta-analysis of the 24 effect values reported in the empirical literature meeting the inclusion criteria, integrated the mean effect size of decentralization reform on the per capita GDP of counties, and conducted heterogeneity test and publication bias test. The results show a moderate correlation between decentralization reform and the per capita GDP of counties, and there is high heterogeneity and publication bias in existing studies, which leads to inconsistencies in empirical evidence and exaggerates the impact of decentralization reform on county economic growth. Therefore, the results of this paper are of great significance for re-examining the economic effects of decentralization reform.
\end{abstract}

Keywords: decentralization reform, economic growth, meta-analysis, heterogeneity, publication bias

\section{INTRODUCTION}

Since the provincial-manager-county reform and the county-power-expansion reform were widely implemented in all Chinese provinces, investigating the economic effects of decentralization reform through the theoretical model of causal inference and econometric tools has always been the focus of academic attention. On the one hand, strengthening the investigation of the economic effects of decentralization reform is conducive to a comprehensive evaluation of the policy effects of decentralization reform, which can provide important decision-making support for deepening the vertical government level reform in practice. On the other hand, identifying the net impact of decentralization on county economic growth through causal inference can provide reliable empirical evidence for testing decentralization theory and its theoretical inference. Therefore, a large number of empirical studies have been carried out around the theme of the provincialmanager-county reform, the county-power-expansion reform, and economic growth. According to the results of empirical research reports, although decentralization theory provides a critical theoretical perspective to explain China's rapid economic growth after the reform and opening up, whether decentralization itself can significantly promote economic growth has not been unanimously supported by empirical evidence. Some studies have proved that decentralization can promote economic growth by stimulating investment in reform counties, but some empirical studies have reported the opposite results, and the empirical evidence in this field shows strong inconsistency.
Can decentralization reform significantly boost economic growth? What is the mean effect size of decentralization on economic growth? Is there heterogeneity and publication bias in empirical studies? All these problems depend on further discussion through meta-analysis. Given this, in order to integrate the effects of decentralization reform on county economic growth, and systematically examine the heterogeneity and publication bias of existing studies, this paper will conduct a meta-analysis on the empirical literature that meets the predetermined inclusion criteria. Meta-analysis is a statistical analysis method that aims to collect and collate all the literature that meets the predetermined inclusion criteria, identify and reduce bias through systematic tools, and thus provide precise and reliable empirical evidence for a research question. In the field of decentralization reform and economic growth, no scholars have conducted a meta-analysis of existing empirical studies, so this paper intends to contribute to this field.

\section{LITERATURE REVIEW}

Many scholars have carried out in-depth empirical research on how decentralization reform affects county economic development. Many scholars reported the positive effect of decentralization reform on county economic growth. Fan and Wang (2013)[1] used the DID method to test the impact of the county-power-expansion reform in Zhejiang Province on local economic growth, and their research results showed that the reform effectively promoted the local economic growth. Hu (2016)[2] used the DID method to test the impact of the provincial-manager-county reform in Henan Province on counties' economic development, and the result showed that the reform could significantly 
promote the economic growth of counties. Ye and Zheng (2013)[3] tested the relationship between the provincialmanager-county reform in Sichuan Province and the economic development of reformed counties based on panel data from 2003 to 2010 . The results showed that the implementation of the provincial-manager-county reform would have a positive impact on the economic development of reformed counties. In addition, Ye (2017) [4]and Liu (2018)[5] also reported the significant positive effect of decentralization reform on county economic growth. However, some pieces of literature have reported the opposite result, that is, decentralization reform will significantly hinder the economic growth of counties. Wen (2016)[6] investigated the impact of decentralization reform on the county economy based on a sample of Anhui Province. The results showed that the provincial-managercounty reform did not promote the economic growth of reformed counties but inhibited counties' economic development to a certain extent. Li (2015)[7] used the panel data model to analyze the data of 78 counties in Shaanxi Province and found a significant negative correlation between the implementation of the provincialmanager-county reform and the economic growth of counties in Shaanxi Province. Zhang (2013)[8] showed that the provincial-manager-county reform in Shandong province hindered the economic growth of counties. In addition, $\mathrm{Wu}$ (2014)[9] also reported that decentralization reform hindered county economic growth. On the whole, scholars have not reached a relatively consistent conclusion on how the decentralization reform affects the development of the county economy.

The inconsistency of empirical evidence indicates that existing empirical studies may be affected by the following three reasons. First, since the estimator of the regression coefficient is a sampling distribution. Therefore, even if decentralization reform has a positive effect on county economic growth, a single empirical study may identify an insignificant or even opposite effect, which leads to the inconsistency of empirical evidence. Second, due to pervious empirical studies conducted by various sample, different regional economic development conditions can lead to different real effects of decentralization reform on county economic growth. In other words, in different regions, the effect of decentralization reform on economic development is different, and there may be high heterogeneity among the existing empirical evidence. Third, due to the preference of journal editors and reviewers for statistically significant regression coefficients, those studies reporting statistically significant regression coefficients are more likely to be published, so existing studies may have publication bias. Due to publication bias, papers reporting that decentralization reform significantly promotes or inhibits county economic development are more likely to be adopted by journals, while papers with insignificant results are less likely to be published, which to some extent exaggerates the inconsistencies of existing studies.

\section{META-ANALYTICAL PROCEDURE}

We carried out the meta-analysis according to the following procedures. First, we determined the literature inclusion criteria, including selecting effect indicators, literature retrieval strategies, and literature screening procedures. Secondly, we coded the pieces of literature included in the meta-analysis and extracted the data required for the meta-analysis. Finally, we use CMA2.0 software to conduct mean effect analysis, heterogeneity test, and publication bias test.

\subsection{Literature Inclusion Criteria}

Specific effect types and indicators should be defined before meta-analysis. Here, we will carry out a metaanalysis of the impact of decentralization reform on the per capita GDP of the reformed counties. The magnitude and direction of the impact are expressed by the regression coefficient before the explanatory variables in the original literature. However, the regression coefficient can not be directly used in the meta-analysis because of dimension inconsistency. Referring to the study of Stanley and Jarrell (1989)[10], we used partial correlation coefficients (PCC) as effect size indicators, which could be calculated by $\mathrm{T}$ values and degrees of freedom of regression coefficients in the original study. Partial correlation coefficients (PCC) are calculated as shown in Formula (1), where $t$ is the $T$ value and $\mathrm{df}$ is the degree of freedom. If the $t$ value is not reported in the original studies, it can be calculated by the value of the regression coefficient and the standard error of the $T$ value. The calculation formula is shown in Formula (2), where $\beta$ is the value of the regression coefficient and $\mathrm{SE}_{\mathrm{t}}$ is the standard error of the $\mathrm{T}$ value.

$$
\begin{gathered}
p c c=t /\left(t^{2}+d f\right)^{1 / 2} \\
t=\beta / S E_{t}
\end{gathered}
$$

We will search and screen the literature according to the following criteria.

- We conducted a literature search in Chinese and English databases with decentralization reform, administrative decentralization, province leading counties, province managing county, county power expansion, enlarging authority, and strengthening counties reform as the keywords, and obtained preliminary literature.

- Only empirical research literature is included, excluding theoretical, strategic, and other types of literature.

- Only those empirical literature with per capita GDP at the county level as the explained variable are included, and pieces of literature with other indicator types are excluded. 
- Data required for meta-analysis should be fully reported in the sample literature, and incomplete data reports should be excluded.

After the above processing process, we initially obtained the sample literature, and coded the sample literature, and then extracted the sample size, regression coefficient, $\mathrm{T}$ value, and degree of freedom in the literature.

\subsection{Data Processing}

After obtaining the data required for the meta-analysis, we carried out the meta-analysis as follows.

- Mean effect analysis. The mean effect reflects the average impact of decentralization reform on per capita GDP. We estimate the mean effect size using both the fixed effect model and the random effect model, report the $95 \%$ confidence interval of the estimated mean effect size, and perform the Z-test of whether the mean effect size is significant 0 .

- Heterogeneity test. Heterogeneity describes the difference of real effects among all individual studies in the meta-analysis. Q test and $\mathrm{I}^{2}$ test are used in this paper to test the heterogeneity of existing studies. Q-value follows the Chi-square distribution of k-1 degrees of freedom. $\mathrm{I}^{2}$ indicates the degree of heterogeneity.

- Publication bias test. Publication bias describes the influence of journal editors and reviewers' preference for significant results on empirical evidence. This paper uses a funnel plot to identify Publication bias. If the effect values in the funnel plot do not show symmetrical distribution, publication bias can be considered; otherwise, there is no publication bias.

\section{RESULTS}

After literature retrieval and screening, we extracted a total of 24 estimated results from the original literature that met the predetermined inclusion criteria. The results of the mean effect test, heterogeneity test, and publication bias test will be reported and analyzed in the following sections.

\subsection{Mean Effect Size}

After integrating 24 effect values through the fixed-effect model and random effect model, we obtained the mean effect size of decentralization reform on the per capita GDP of counties. The results of the mean effect test are shown in Table 1.

Under the fixed effects model, the mean effect size of decentralization reform on the per capita GDP level at the county level is 0.173 , and its $95 \%$ confidence interval is $[0.156,0.191]$, and the $Z$ value is significantly not 0 at the significance level of 0.001 . Under the random effect model, the value of the mean effect size between decentralization reform and per capita GDP is 0.215 , and its $95 \%$ confidence interval is $[0.135,0.292]$, and the $\mathrm{Z}$ value is significantly not 0 at the significance level of 0.001. This result means that, without considering publication bias, decentralization reform increased the per capita GDP of reformed counties by about 0.2 units compared with non-reform counties, and the growth effect is significant at the significance level of $1 \%$. According to Lipsey and Wilson's (2001) [11] division of correlation degree, when the correlation coefficient is between 0.1 and 0.4 , it is a moderate correlation. According to this standard, there is a moderate correlation between decentralization reform and the per capita GDP of reformed counties.

Table 1. Results of mean effects test

\begin{tabular}{|c|c|c|c|c|c|}
\hline \multirow{2}{*}{ Model } & \multirow{2}{*}{ Number effects } & \multicolumn{3}{|c|}{ Effects and 95\% interval } & \multicolumn{2}{c|}{ Test of null(2-Tail) } \\
\cline { 3 - 6 } & & Point estimate & Lower limit & Upper limit & Z-Value \\
\hline Fixed & 24 & 0.173 & 0.156 & 0.191 & $19.042^{* * * * *}$ \\
\hline Random & 24 & 0.215 & 0.135 & 0.292 & $5.209^{* * * *}$ \\
\hline
\end{tabular}

\subsection{Heterogeneity Test}

$\mathrm{Q}$ test and $\mathrm{I}^{2}$ test were used in this paper to analyze heterogeneity among different pieces of literature, and the results are shown in Table 2. The Q value is 451.597 and significant at the level of $1 \%$, suggesting high heterogeneity in the existing studies. The $\mathrm{I}^{2}$ test result was 94.907, indicating that the proportion of effect difference caused by heterogeneity accounted for $94.907 \%$ of the total variation of effect size. According to Higgins's (2003) [12] classification of the degree of heterogeneity, high heterogeneity exists in existing empirical evidence when the $\mathrm{I}^{2}$ value is greater than $75 \%$. According to this classification standard, there is a high degree of heterogeneity in existing studies.

Table 2. results of heterogeneity test

\begin{tabular}{|c|c|c|}
\hline Q-Value & df(Q) & I-squared \\
\hline 451.597 & 23 & $94.907^{* * * *}$ \\
\hline \multicolumn{3}{|c|}{} \\
${ }^{* * *}: \mathrm{P}<0.001$
\end{tabular}

\subsection{Publication Bias}

In this study, a funnel plot was used to test publication bias in existing studies, and the results are shown in Fig. 1. As can be seen from the figure, the effect values included in 
the meta-analysis presented a trend of right bias on the whole, so there is publication bias in the existing empirical studies. The existence of publication bias indicates that, in the field of decentralization reform and county economic growth, those results that show that decentralization reform significantly promotes per capita GDP are more likely to be published, which may be due to the preference of journal editors, external review experts or authors themselves for significant results. The existence of publication bias also indicates that the existing empirical evidence may exaggerate the relationship between decentralization reform and the per capita GDP of counties, and we must carefully view the economic effects brought by decentralization reform.

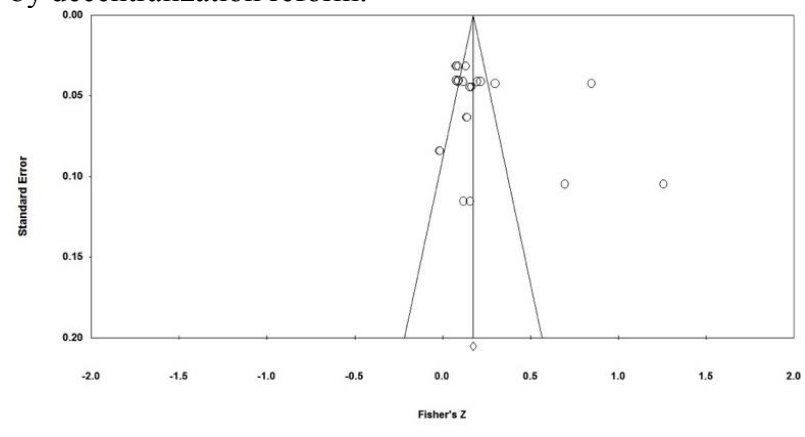

Figure 1. Funnel plot of the standard error of Fisher's Z.

\section{CONCLUSION}

Whether decentralization reform can promote the economic growth of the reformed counties has always been the focus of academic attention, but empirical evidence fails to provide a relatively consistent conclusion. In this paper, we conducted a meta-analysis of 24 effect sizes reported in literature meeting the inclusion criteria, integrated the effects of decentralization reform on GDP per capita of counties, and conducted a heterogeneity test and publication bias test. The results show a moderate positive correlation between decentralization reform and the per capita GDP of counties, and the reform increased the per capita GDP of counties by about 0.2 units. However, the high degree of heterogeneity in the existing empirical studies is the main reason for the inconsistency of the empirical results. At the same time, there is publication bias in the existing empirical studies, which means that the existing empirical evidence exaggerates the impact of decentralization reform on the per capita GDP of counties. Thereby, this study attaches importance to the economic effects brought by decentralization reform.

\section{REFERENCES}

[1] Fan Yong, Wang Wei. A Comparative study on the Reform effect of county-power-expansion: A case study of Zhejiang Province [J]. Journal of Public Management, 2013,10(01): pp. 10-18. (In Chinese).
[2] Hu Yalan. The influence of the system reform of provincial-manager-county on the development of county economy in Henan Province[J]. Finance and Accounting Monthly, 2016(12): pp.44-48. (In Chinese).

[3] Ye Zirong, Zheng Rongsheng. An empirical study on the economic performance of the reform of provincial-manager-county: Empirical evidence from the panel data of counties (cities) in Sichuan Province[J]. New Horizons from Tianfu, 2013, 05: pp.51-54. (In Chinese).

[4] Ye Nian. Study on the Province Directly Managing County System Reform in Hubei[D]. Wuhan University, 2013. (In Chinese).

[5] Liu Xiaoqian. The impact of County Administrated by Province reform on counties' economic performance and people's livehood: Take Sichuan Province as example[D]. Southwest Jiaotong University, 2018. (In Chinese).

[6] Wen Yanbing. Gains and Losses of County Directly Administrated by Province Reform: Empirical Studies Based on DID [J]. Yuejiang Academic Journal, 2016, 8(03): pp. 38-51. (In Chinese).

[7] Li Miaomiao. The empirical research of provincegoverning-county fiscal system reform and performance of Shaanxi province[D]. Xi'an University of Architecture and Technology, 2015. (In Chinese).

[8] Zhang Wenwen. Study on the influence of provincial direct county reform on county economic development [D]. Shandong University of Finance and Economics, 2013 (In Chinese).

[9] Wu Gan. Study on the impacts of the Reform about Direct Administration between Province and County Financial System in Henan on economic growth and financial self-sufficiency rate[D]. Xiamen University, 2014. (In Chinese).

[10] T D Stanley, S B Jarrell. Meta-Regression Analysis: A Quantitative Method of Literature Surveys. Journal of Economic Surveys, 1989, 3(2): pp. 161-170.

[11] M W Lipsey, D B Wilson. Practical meta-analysis. California: Sage, 2001.

[12] J P T Higgins, S G Thompson, J J Deeks, D G Altman. Measuring inconsistency in meta-analyses. British Medical Journal, 2003 (7414): pp. 557-560. 